\title{
OTIMIZAÇÃO OPERACIONAL DO PROCESSO DE CRISTALIZAÇÃO DA SACAROSE EM LEITO VIBRADO
}

\author{
F. H. F. CALDAS ${ }^{1}$, D. B. QUINTINO ${ }^{1}$, e R. A. MALAGONI ${ }^{1}$ \\ ${ }^{1}$ Universidade Federal de Uberlândia, Faculdade de Engenharia Química \\ E-mail para contato: malagoni@ feq.ufu.br
}

\begin{abstract}
RESUMO - O processo de cristalização é de suma importância na produção industrial de diversos compostos, incluindo a indústria alimentícia, e também é considerado um método de baixo custo na produção de sólidos puros. A cristalização é realizada mediante dois processos básicos, nucleação, que está relacionada à formação de novos núcleos e crescimento do cristal. O objetivo deste trabalho consistiu em analisar a produtividade percentual, dimensão e etapas de crescimento de cristais de sacarose obtidos em um cristalizador de leito vibrado. Soluções supersaturadas de sacarose foram preparadas e transferidas ao cristalizador, este operando com número constante de sementes. Na determinação da condição otimizada de operação, foram consideradas as seguintes variáveis: supersaturação, intensidade de vibração e tempo de operação. Após o processo de cristalização, os cristais foram separados, lavados e secos para determinação da produtividade em termos percentuais e o tamanho final dos mesmos. Observou-se a maior influência da variável supersaturação na cristalização de sacarose para obter-se cristais maiores e com maior produtividade, a variável intensidade de agitação seguiu com importância secundária. $\mathrm{Na}$ análise de crescimento dos cristais foram selecionadas amostras em intervalos de 14 minutos e analisadas as áreas superficiais em um estereomicroscópio e, posteriormente, realizou-se tratamento dos resultados com softwares Image J e Statistica.
\end{abstract}

\section{INTRODUÇÃO}

A operação unitária de cristalização é um dos métodos de produção de material particulado com elevada pureza. Esta operação proporcionou um desenvolvimento contínuo das indústrias de processos químicos, que visam obter produtos de alta qualidade para atender a demanda do mercado interno e externo (Malagoni, 2010).

O processo de cristalização se desenvolve por dois mecanismos simultâneos principais: a nucleação e o crescimento dos cristais, que juntos com fenômenos secundários vão determinar a distribuição granulométrica dos cristais, caracterizada por um tamanho médio e sua dispersão (Derenzo, 2003).

O objetivo geral deste trabalho constituiu em promover um método de cristalização da sacarose em leito vibrado através de um Planejamento Composto Central (PCC), com a finalidade de obter a condição ótima do processo, a fim de maximizar a produção de sacarose e análise da variação de dimensão do cristal durante o processo de cristalização. 


\section{MATERIAL E MÉTODOS}

A unidade experimental utilizada neste estudo encontra-se montada no Laboratório de Cristalização da Faculdade de Engenharia Química na Universidade Federal de Uberlândia. A Figura 1 (A) apresenta os equipamentos que a compõe, assim como os detalhes do sistema de vibração (B).
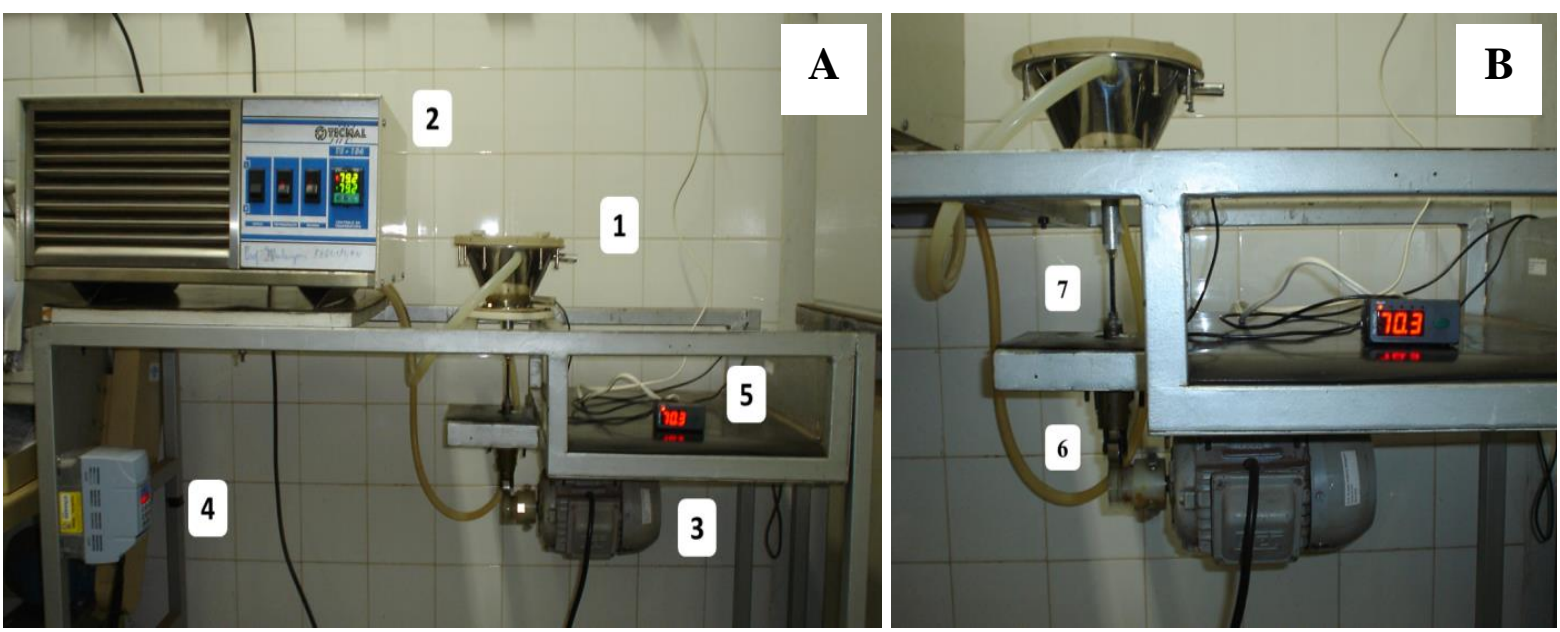

Figura 1 - (A) Equipamentos utlizados nos experimentos; (B) sistema de vibração.

Os equipamentos mostrados na Figura 1 compõe a unidade, são eles: 1 - Cristalizador; 2 - Banho termostatizado (Tecnal, modelo TE 184); 3 - Motor elétrico (WEG, 0,75 CV); 4 Inversor de frequência (WEG, modelo CFW 08); 5 - Indicador de temperatura da solução no interior do cristalizador; 6 - Excêntrico e 7 - Eixo vibratório.

O cristalizador utilizado é confeccionado em aço inoxidável, é encamisado e possui configuração tronco-cônico com angulação de $65^{\circ} \mathrm{com}$ a horizontal. A vedação do cristalizador é realizada por uma borracha flexível, própria para utilização em alimentos. As dimensões do aparelho e sua exata configuração podem ser encontradas em Malagoni (2010).

\subsection{Procedimento Experimental}

Sacarose comercial $\left(\right.$ Caeté $^{\circledR}$ ) foi usada para preparar soluções de sacarose, conforme o planejamento de experimentos. A solução foi preparada em banho-maria e os dados de solubilidade de sacarose em água foram retirados de Oetterer (2006). Após a solubilização da amostra, $280 \mathrm{~mL}$ da solução foram transferidos ao cristalizador que foi previamente montado e aquecido à temperatura de $358,2 \mathrm{~K}$. Realizada a transferência, a temperatura de solução foi reduzida gradativamente até $343,2 \mathrm{~K}$, sendo esta a temperatura de operação do processo.

Estabilizado o sistema na temperatura de operação, fez-se a adição de $12 \mathrm{~g}$ de sementes de açúcar cristal previamente selecionados através do peneiramento da sacarose comercial em um vibrador (Retsch, modelo AS-200) por $0,5 \mathrm{~h}$, com amplitude de $1,5 \mathrm{~mm}$, no qual as sementes utilizadas foram obtidas na peneira Tyler $20(0,841 \mathrm{~mm})$. As sementes foram lavadas com uma solução de sacarose saturada a 298,2 K, estas possuíam valor médio de $0,523 \cdot 10^{-3} \mathrm{~m}$, número obtido através da média de comprimento de 50 sementes de sacarose, 
medidas com paquímetro digital (King Tools, resolução 0,01mm). Após a introdução dos cristais utilizados como sementes, iniciou-se a contagem do tempo de cristalização.

Ao findar a cristalização, todo material contido no cristalizador foi retirado e despejado sobre uma peneira de malha Tyler $24(0,707 \mathrm{~mm})$ previamente aquecida a uma temperatura de 333,2 K, separando os cristais da solução de sacarose remanescente. Concomitantemente, os cristais foram lavados em solução alcoólica de $75 \%$ e $96 \%$, conforme analisado por Pereira (1997). Após a separação, os cristais foram secos ao permanecer na estufa por $48 \mathrm{~h}$ a temperatura de $333,2 \mathrm{~K}$.

\subsection{Variáveis utilizadas e respostas analisadas}

$\mathrm{Na}$ análise de resultados experimentais, estudou-se a influência das seguintes variáveis do processo: supersaturação, intensidade de agitação e tempo de operação. Os resultados obtidos experimentalmente foram analisados através da produtividade alcançada e do diâmetro médio de Sauter. A supersaturação da solução de sacarose foi calculada através da Equação 1, em que $S$ representa o grau de supersaturação, $C$ a concentração do sistema na temperatura de operação e $C^{*}$ a concentração de saturação na temperatura do sistema, ambas as concentrações em $\mathrm{kg} / \mathrm{kg}$ de água.

$$
S=\frac{C}{C^{*}}
$$

A intensidade de vibração foi determinada pelo número adimensional de vibração, como mostra a Equação 2, em que $A$ e $\omega$ são respectivamente, a amplitude de vibração (m) e a frequência angular de vibração $\left(1 . \mathrm{s}^{-1}\right)$ do agente excitador externo que promove a vibração no leito, e $g$ representa a aceleração da gravidade no local.

$$
\Gamma=\frac{A \omega^{2}}{g}
$$

O valor da frequência angular foi determinado através da Equação 3.

$$
\omega=v \frac{2 \pi}{60}
$$

$\mathrm{Na}$ Equação 3, $v$ representa a velocidade do excêntrico em rpm fornecida pelo tacômetro digital (Shimpo, modelo DT-205B). A Equação 4 apresenta como foi realizado o cálculo da produtividade.

$$
y(\%)=\left(\frac{m_{c}}{m_{s}}-1\right) .100
$$

sendo: $y(\%)$ a produtividade em termos percentuais, $m_{\mathrm{c}}$ a massa de cristais obtida ao final do processo de cristalização $(\mathrm{g})$ e $m_{\mathrm{s}}$ a massa de sementes $(\mathrm{g})$ inoculadas no cristalizador no 
início do processo de cristalização. O diâmetro médio de Sauter $\left(\bar{D}_{s t}\right)$ foi obtido através da Equação 5, esta resposta relaciona a fração mássica de sementes retidas $\left(\Delta x_{\mathrm{i}}\right)$ com o diâmetro médio da porção retida $\left(D_{\mathrm{i}}\right)$ em mm, este último, é obtido através da média entre os tamanhos da peneira na qual os cristais foram retidos e da peneira imediatamente superior a esta.

$$
\bar{D}_{s t}=\frac{1}{\sum_{i=1}^{p} \frac{\Delta x_{i}}{D_{i}}}
$$

\subsection{Planejamento de Experimentos}

O Planejamento Composto Central (PCC) é um planejamento fatorial de primeira ordem acrescido de pontos adicionais a fim de permitir a estimativa dos parâmetros de superfícies de segunda ordem. Através do planejamento de experimentos elaborado com auxílio do software Statistica, foi possível o estudo da influência de variáveis no processo de cristalização.

Neste estudo, o PCC foi constituído por três experimentos em condições operacionais em valores intermediários e idênticos, conhecidos como pontos centrais, correspondendo aos três últimos experimentos 15,16 e 17 . Tal repetição foi realizada com o intuito de verificar variação nos resultados e análise de existência de erro sistemático na ocorrência de divergência das respostas. O planejamento ainda foi constituído de seis experimentos nos pontos axiais $(\alpha)$, isto é, pontos nos quais uma das variáveis analisadas operou em condição extrema. As variáveis codificadas $x_{1}, x_{2}$ e $x_{3}$ estão respectivamente relacionadas com a supersaturação, grau de vibração e tempo de operação e são calculadas através das Equações 6,7 e 8.

$$
\begin{aligned}
& x_{1}=\frac{S-1,11}{0,06} \\
& x_{2}=\frac{\Gamma-0,76}{0,33} \\
& x_{3}=\frac{t-1,25}{0,42}
\end{aligned}
$$

O valor utilizado para este nível no planejamento foi o $(\alpha)$ de ortogonalidade, com valor igual a 1,353, este também obtido pelo software Statisica. A matriz codificada com os 17 experimentos é apresentada junto com os resultados na Seção 3.

\subsection{Análise de Imagem}

Após tratamento de dados dos resultados obtidos experimentalmente, com o auxílio do software Statistica, foi determinada a condição otimizada para a região de estudo considerada. Inicialmente foi proposta a análise da cinética de cristalização da sacarose utilizando-se técnica de refração a laser no equipamento Mastersize. Os cristais obtidos experimentalmente 
excedem o tamanho máximo no qual o aparelho consegue analisar, consequentemente, não foi possível realizar tal análise tanto em via seca quanto em via úmida.

Como alternativa, durante o processo de cristalização, foram coletadas amostras no intervalo de 14 minutos, estas, posteriormente analisadas no estereomicroscópio (Nikon, modelo SMZ800) e no software Image J comparando a média da área do cristal em cada imagem.

\section{RESULTADOS E DISCUSSÃO}

Os resultados dos experimentos para as respostas diâmetro de Sauter e produtividade podem ser visualizados na Tabela 1 . Nos resultados apresentados, o tamanho médio dos cristais obtidos experimentalmente foi de $1,186 \mathrm{~mm}$ e o valor médio para resposta produtividade foi $109,18 \%$. Nota-se que para experimentos que utilizaram maiores valores de supersaturação e tempos intermediário, obteve-se melhores resultados, como nos experimentos 2 e 10 .

Tabela 1 - Resultados experimentais obtidos pelo PCC

\begin{tabular}{|c|c|c|c|c|c|}
\hline Experimento & $x_{1}$ & $x_{2}$ & $x_{3}$ & $y(\%)$ & $\bar{D}_{s t}(\mathrm{~mm})$ \\
\hline 1 & $-1,000$ & $-1,000$ & $-1,000$ & 61,1667 & 1,11704 \\
\hline 2 & $+1,000$ & $-1,000$ & $-1,000$ & 162,5099 & 1,34053 \\
\hline 3 & $-1,000$ & $+1,000$ & $-1,000$ & 94,7500 & 1,23829 \\
\hline 4 & $+1,000$ & $+1,000$ & $-1,000$ & 151,5833 & 1,26256 \\
\hline 5 & $-1,000$ & $-1,000$ & $+1,000$ & 93,5000 & 1,05696 \\
\hline 6 & $+1,000$ & $-1,000$ & $+1,000$ & 129,9166 & 1,33906 \\
\hline 7 & $-1,000$ & $+1,000$ & $+1,000$ & 122,3333 & 1,10638 \\
\hline 8 & $+1,000$ & $+1,000$ & $+1,000$ & 98,3333 & 1,23269 \\
\hline 9 & $-1,353$ & 0,000 & 0,000 & 117,8500 & 1,12597 \\
\hline 10 & $+1,353$ & 0,000 & 0,000 & 158,6667 & 1,46570 \\
\hline 11 & 0,000 & $-1,353$ & 0,000 & 77,2500 & 1,29269 \\
\hline 12 & 0,000 & $+1,353$ & 0,000 & 84,8500 & 1,15672 \\
\hline 13 & 0,000 & 0,000 & $-1,353$ & 98,3333 & 1,10280 \\
\hline 14 & 0,000 & 0,000 & $+1,353$ & 131,4167 & 1,25882 \\
\hline 15 & 0,000 & 0,000 & 0,000 & 92,6667 & 1,13028 \\
\hline 16 & 0,000 & 0,000 & 0,000 & 89,4167 & 1,17706 \\
\hline 17 & 0,000 & 0,000 & 0,000 & 91,5000 & 1,14871 \\
\hline
\end{tabular}

As Figuras 2 e 3 mostram as superfícies de resposta para produtividade e diâmetro de Sauter em função das variáveis analisadas em níveis codificados, obtidas nas Equações 6, 7 e 8. Na superfície de resposta da Figura 2, observa-se que a maximização da produtividade nesta faixa de estudo é alcançada com o aumento da supersaturação e utilização de menores tempos de cristalização. 


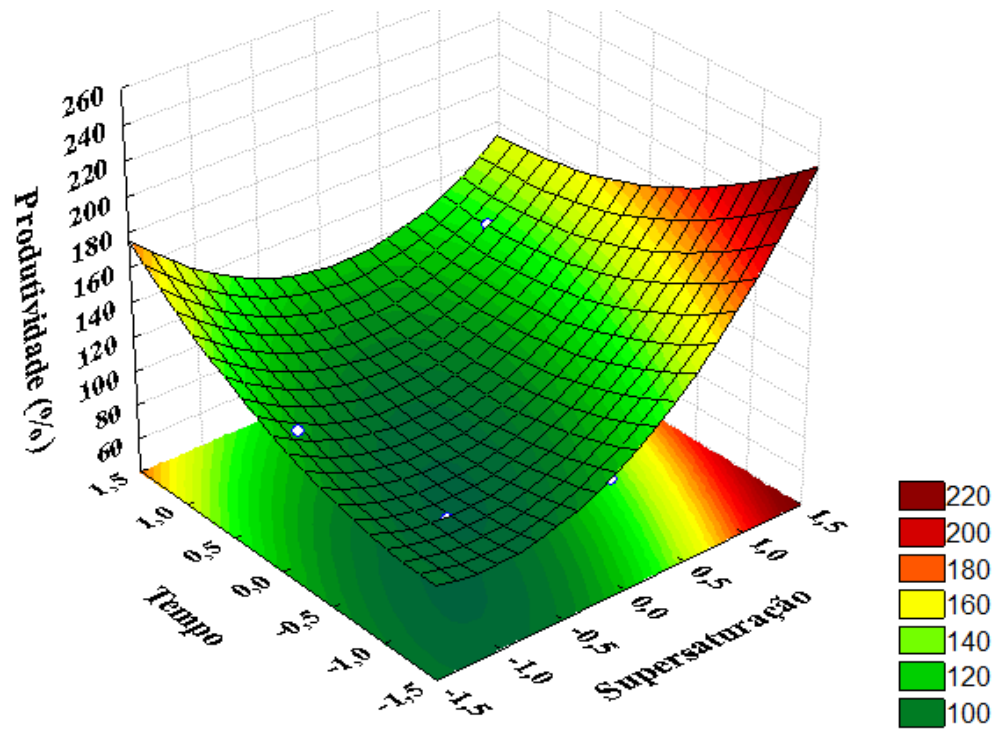

Figura 2 - Superfície de resposta da produtividade em função das variáveis tempo e supersaturação.

Para a resposta de Diâmetro de Sauter, nota-se na Figura 3, que as melhores respostas são alcançadas com menor intensidade de agitação e maior nível de supersaturação.

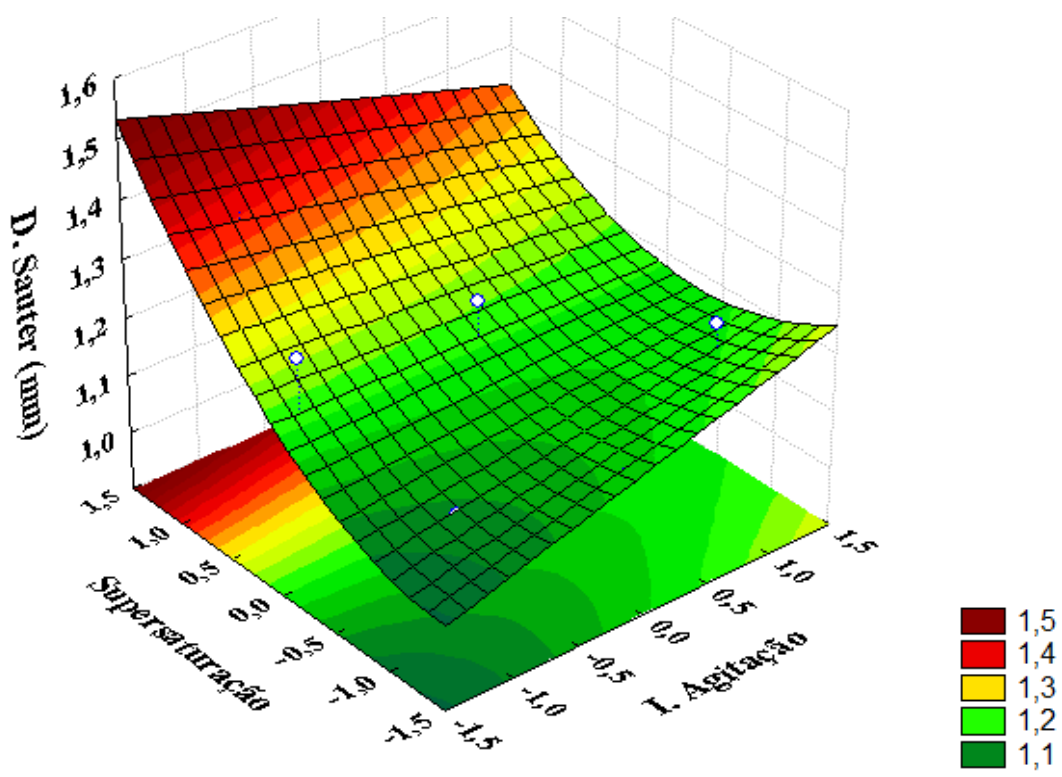

Figura 3 - Superfície de reposta para diâmetro de Sauter em função das variáveis supersaturação e intensidade de agitação.

A análise crescimento do cristal foi realizada na condição otimizada obtido pelo software Statistica, tal condição operacional sugere os seguintes níveis codificados para cada variável analisada: $+1,353$ para supersaturação, $-0,731$ para intensidade de agitação, $+0,217$ para tempo de operação, isto é, $S=1,20, \Gamma=0,518$ e $t=82 \mathrm{~min}$. Foram realizados testes em triplicada, a fim de se verificar a exatidão do modelo proposto. 
Os experimentos apresentaram em média diâmetro médio de Sauter de 1,374 mm, com reposta prevista de 1,467 mm. Para produtividade, os experimentos na condição otimizada obtiveram valor médio de $159,21 \%$, o modelo previu o valor de $162,64 \%$. Nesta condição, o tempo ótimo de operação proposto foi de 82 minutos. As equações de modelo para as duas respostas analisadas são apresentadas, a Equação 9 é relativa à produtividade alcançada e a Equação 10 para a resposta Diâmetro de Sauter.

$$
\begin{aligned}
& 94,21+43,897 x_{1}{ }^{2}+38,739 x_{1}-18,604 x_{2}{ }^{2}-18,351 x_{3}{ }^{2}-26,23 x_{1} x_{2}-36,4 x_{1} x_{3} \\
& 1,174+0,051 x_{1}{ }^{2}+0,096 x_{1}-0,044 x_{1} x_{2}
\end{aligned}
$$

Nas Equações 9 e 10, as variáveis $x_{1}, x_{2}$ e $x_{3}$ são respectivamente supersaturação, intensidade de agitação e tempo de operação. O nível de significância estipulado para os ajustes foi de $10 \%$. Nota-se que no primeiro ajuste a variável $x_{1}$ apresenta alta influência na produtividade e que a variável $x_{2}$ não apresenta coeficiente de regressão quadrático, neste ajuste o coeficiente de correlação quadrático $\left(R^{2}\right)$ foi de 0,9229 . Para Equação 10, somente as variáveis $x_{1}$ e $x_{2}$ apresentam influência significativa dentro das regiões do estudo, neste ajuste o coeficiente de correlação quadrático $\left(\mathrm{R}^{2}\right)$ foi de 0,7208 . Na Tabela 2 visualizam-se os resultados obtidos no tratamento de imagens das amostras retidas durante o processo de cristalização.

Tabela 2 - Área média das imagens dos cristais durante o processo de cristalização

\begin{tabular}{|c|c|c|}
\hline$t$ (minutos) & $A\left(\mathrm{~mm}^{2}\right)$ & $\Delta A\left(\mathrm{~mm}^{2}\right)$ \\
\hline 14 & 1,083 & 0,245 \\
\hline 28 & 1.328 & 0,218 \\
\hline 42 & 1,546 & 0,046 \\
\hline 56 & 1,592 & 0,036 \\
\hline 70 & 1,628 & 0,072 \\
\hline 82 & 1,700 & \\
\hline
\end{tabular}

A diferença entre áreas de cada intervalo teve significativa queda após o vigésimo oitavo minuto de operação. Durante todo o processo de coleta de amostras e tratamento de imagens, notou-se a presença de cristais com dimensões inferiores à dimensão dos cristais utilizados como sementes, sendo estes provenientes de cristalização de segunda ordem ou devido à quebra ocasionada pelo choque entre os próprios cristais ou entre estes e a parede do cristalizador. A Figura 4 mostra imagens de amostras distintas, coletadas em três diferentes tempos.
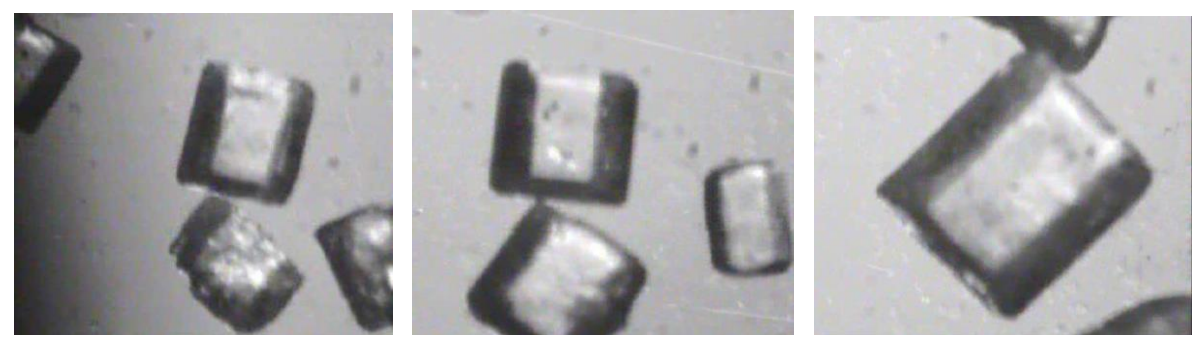

Figura 4 - Imagem de diferentes cristais nos tempos de 14, 56 e 82 minutos, respectivamente. 


\section{CONCLUSÃO}

Neste trabalho, concluiu-se que no processo de cristalização de sacarose em leito vibrado, todas as variáveis analisadas influenciaram na maximização de pelo menos uma resposta. Como já esperado, a supersaturação teve influência direta nas reposta, sendo desejado seu maior valor. De acordo com as equações de modelo, a interação entre as variáveis supersaturação e intensidade de vibração deve ser considerada na produtividade e diâmetro médio dos cristais, e a variável tempo e sua interação com a supersaturação não pode ser descartada na produtividade dos cristais. A condição de resposta máxima estabeleceu valor máximo para supersaturação, valor baixo para a intensidade de vibração, e intermediário par o tempo de operação, isto é, $S=1,20, \Gamma=0,518$ e $t=82 \mathrm{~min}$.

Os resultados de imagem obtidos via microscopia também sugerem que a influência da variável supersaturação tem decréscimo durante o processo de cristalização, isto foi constatado pelo fato de que esta mesma variável tem seu valor reduzido com o tempo devido à passagem do soluto presente na solução para o cristal. Em trabalhos futuros, recomenda-se análise da aglomeração de cristais e o estudo usando equipamentos que possam causas agitação e/ou efeitos estruturais em outras escalas de tamanho como, por exemplo, microondas.

\section{REFERÊNCIAS}

DERENZO, S. Cristalização de ácido adípico em diferentes solventes. 2003. Tese (Doutorado em Engenharia Química) - Universidade Federal de São Carlos, São Carlos, 2003.

MALAGONI, R. A. Cristalização de ácido cítrico em leito vibrado. 2010. 297 p. Tese (Doutorado em Engenharia Química) - Universidade Federal de Uberlândia, Uberlândia, 2010.

OETTERER, M; SARMENTO, REGITANO, d’D. M. A; SPOTO, M. H. F. Fundamentos de Ciência e Tecnologia de Alimentos. v.1, p. 135-195, 2006.

PEREIRA, A. G. Cristalização de sacarose em leito vibro - jorrado. 1997. 144 p. Dissertação (Mestrado em Engenharia Química) - Universidade Federal de Uberlândia, Uberlândia, 1997.

\section{AGRADECIMENTOS}

Agradecemos a CAPES e ao CNPq pelas bolsas de Mestrado e Iniciação Científica, respectivamente, à Faculdade de Engenharia Química da Universidade Federal de Uberlândia pela infraestrutura disponibilizada para a realização desta pesquisa no Laboratório de Cristalização. Agradecemos também a Fundação de Amparo à Pesquisa do Estado de Minas Gerais (FAPEMIG) pelos recursos concedidos no Projeto de Participação Coletiva em Eventos Técnicos-Científicos (PCE-00082-14). 